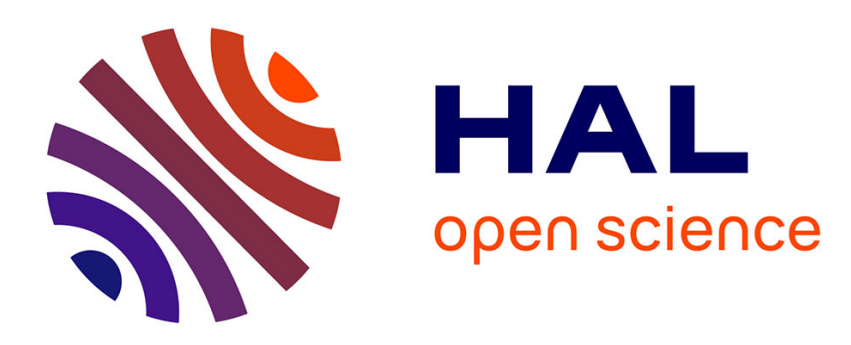

\title{
Étude des interfaces enterrées par diffraction de rayons $\mathrm{X}$
}

\author{
A. Bourret
}

\section{To cite this version:}

A. Bourret. Étude des interfaces enterrées par diffraction de rayons X. Journal de Physique IV

Proceedings, 1997, 07 (C6), pp.C6-19-C6-29. 10.1051/jp4:1997602 . jpa-00255701

\section{HAL Id: jpa-00255701 https://hal.science/jpa-00255701}

Submitted on 1 Jan 1997

HAL is a multi-disciplinary open access archive for the deposit and dissemination of scientific research documents, whether they are published or not. The documents may come from teaching and research institutions in France or abroad, or from public or private research centers.
L'archive ouverte pluridisciplinaire HAL, est destinée au dépôt et à la diffusion de documents scientifiques de niveau recherche, publiés ou non, émanant des établissements d'enseignement et de recherche français ou étrangers, des laboratoires publics ou privés. 


\title{
Étude des interfaces enterrées par diffraction de rayons $\mathbf{X}$
}

\author{
A. Bourret \\ Département de Recherche Fondamentale sur la Matière Condensée, SP2M, CEN Grenoble, \\ 38054 Grenoble cedex 9, France
}

\begin{abstract}
X-ray diffraction (and/or diffusion) is a powerful tool for studying buried interfaces. The experimental X-ray techniques are reviewed, including reflectivity at low angle, extended reflectivity, non-specular diffuse scattering, grazing incidence diffractometry. The main results obtained recently on a variety of interfaces are presented briefly for different couples of solid or liquid materials. Semiconductor-semiconductor and semiconductor-metals were among the most heavily studied : the determination of roughness, steps correlation or localised interfacial structures are available on several systems. The advent of new generation X-ray synchrotron sources opens up new perspectives in this field.
\end{abstract}

\section{INTRODUCTION}

Le besoin d'informations à une échelle atomique dans les matériaux est aujourd'hui partagé par les physiciens, les chimistes de l'état solide aussi bien que par les industriels travaillant sur les matériaux avancés. De plus en plus de secteurs de la recherche sur les matériaux abordent en effet des propriétés qui sont largement dépendantes des arrangements structuraux ou électroniques à une échelle proche de l'atome : les secteurs de la micro-électronique, des revêtements de surface ou des enregistrements magnétiques en sont des exemples précis. Les interfaces jouent dans ces matériaux un rôle considérable pour contrôler les propriétés physiques ou chimiques. Par ailleurs, les résultats de la simulation numérique atomique et les expériences virtuelles qui deviennent ainsi possibles, doivent être sans arrêt comparés aux expériences.

Or il existe peu d'outils expérimentaux pour l'étude des interfaces. La microscopie électronique et la diffraction X sont parmi les seules qui permettent d'accéder à une connaissance des interfaces enterrées. Cette dernière présente l'avantage d'être non destructive et fait l'objet de cet article. Dans une première partie les principes des méthodes de diffraction-diffusion des rayons X seront exposés : ces méthodes diffèrent par la direction du signal analysé. Puis une revue des principaux résultats expérimentaux obtenus ces dernières années sur diffétentes interfaces solide-solide ou solide-liquide sera présentée.

\section{LES MÉTHODES EXPÉRIMENTALES}

Les méthodes de diffraction et/ou diffusion se sont rapidement développées dans la dernière décade en particulier grâce à la construction de sources de rayonnement synchrotron de deuxième génération, c'est à dire ayant des brillances et des flux exceptionnels par rapport aux sources classiques. En particulier la possibilité d'observer in-situ la croissance des couches est particulièrement attractive [1-3]. Ces méthodes ont déjà fait l'objet de nombreuses revues auxquelles nous renvoyons pour plus de détail [4-9]. Nous résumerons ici seulement les principes de ces méthodes particulièrement celles qui enregistrent la diffusion et/ou la diffraction d'un faisceau incident, en général en incidence rasante.

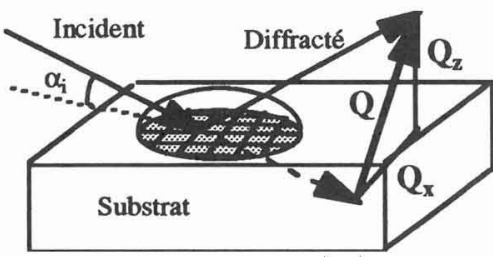

Figure $1:$ a) diagramme schématique de la géométrie de l'interaction de l'échantillon avec le faisceau de rayons $\mathrm{X}$,
La distribution des rayons $X$ diffusés par un objet quelconque s'analyse aisément dans le réseau réciproque avec l'amplitude et la direction du vecteur de diffusion, $\mathbf{Q}$ (Fig. 1). Plusieurs zones du réseau réciproque peuvent être explorées et portent des renseignements complémentaires sur l'objet :

- pour $\mathbf{Q}$ le long de l'axe $\mathrm{Oz}$ il s'agit de la géométrie de la "réflectivité". Ce nom est en général réservé à des angles relativement faibles, mais dans le cas des objets cristallins ayant 
un plan réticulaire parallèle a la surface (ou à l'interface) on rencontre alors des points de diffraction le long de cet axe $\mathrm{Oz}$ et on parle alors de "réflectivité étendue".

- pour $\mathbf{Q}$ très faible et quelconque, il s'agit de la géométrie de la "diffusion aux petits angles"

- pour $\mathbf{Q}$ coïncidant avec un noeud du réseau du substrat ou de la couche déposée ou de l'interface ou de la multicouche lorsque l'un de ceux ci est périodique, il s'agit de la "diffraction"

- pour Q sur la tige perpendiculaire à la surface ou l'interface menée à partir des noeuds du réseau réciproque, il s'agit de la "tige de troncature".

- pour $\mathbf{Q}$ quelconque et dans aucune catégorie précedente, il s'agit de la "diffusion diffuse"

La mesure de l'une ou de l'autre portion du réseau réciproque (Fig. 2) doit s'adapter aux échantillons à étudier, la tendance générale étant d'employer plusieurs types de mesure sur le même objet pour une description plus complète de sa structure.

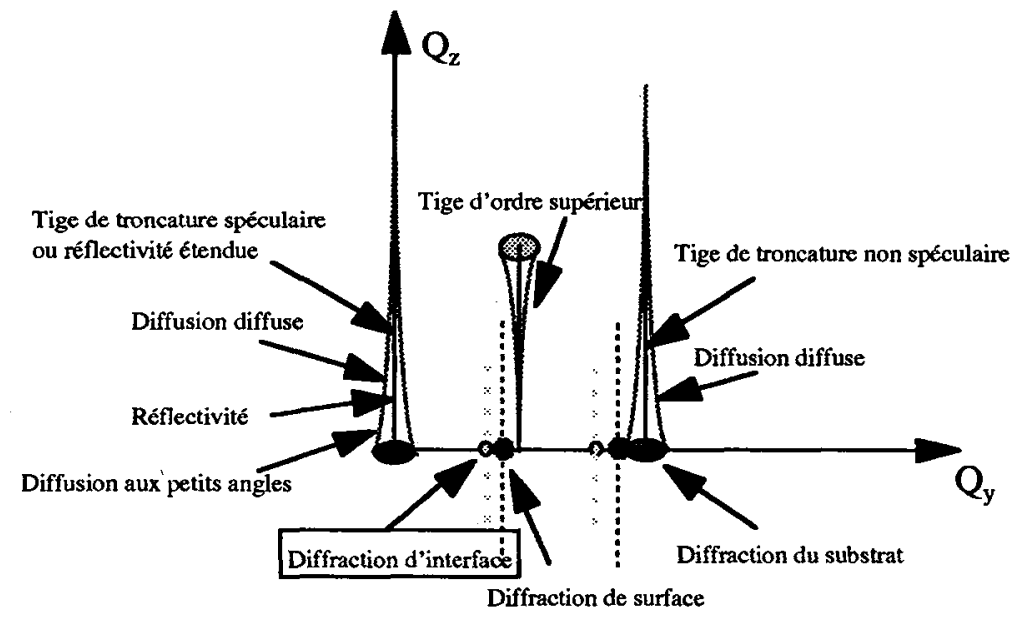

Figure 2 : Les différentes parties du réseau réciproque explorées au cours d'une expérience de diffraction et/ou diffusion de rayons $\mathrm{X}$

\subsection{L'incidence rasante}

La géométrie généralement adoptée pour l'étude des interfaces enterrées au voisinage d'une surface est celle de l'incidence rasante du faisceau incident. L'angle d'incidence, $\alpha_{i}$, définit la profondeur de pénétration dans le matériau. Pour un angle inférieur à l'angle critique, $\theta_{\mathcal{c}}$, la pénétration est très faible et de l'ordre de grandeur de quelques nanomètres $(2 \mathrm{à} 5 \mathrm{~nm})$. Au dessus de cette valeur la pénétration varie très rapidement : elle est déjà de quelques centaines de nanomètres à 1,3 fois l'angle critique. Deux conséquences découlent de cette variation rapide : i) la surface de l'échantillon étudié doit être rigoureusement plane pour que l'angle d'incidence soit bien défini car l'angle d'incidence est de l'ordre de $10^{-4}$ à $10^{-3}$ radians et ii) la divergence du faisceau incident doit être très inférieure à l'angle critique. Ces conditions sont particulièrement bien satisfaites sur les sources synchrotron, en particulier sur les éléments d'insertion dont la brillance est élevée.

\subsection{La réflectivité}

La mesure de la réflectivité des rayons $X$ (angle d'incidence égal à l'angle d'émergence dans la géométrie dite "spéculaire") est une méthode très ancienne qui a été appliquée pour l'étude du voisinage des surfaces [10]. Dans le cas d'un milieu semi-infini homogène la courbe de réflectivité dite de Fresnel met en évidence l'angle critique en dessous duquel il y a réflexion totale. Dans le cas d'un milieu semi-infini inhomogène ou stratifié elle donne des renseignements précis sur le profil en profondeur de la densité électronique à partir de la surface sur les premiers $100 \mathrm{~nm}$ environ [11]. Cette méthode a été largement utilisée également pour quantifier la rugosité moyenne des interfaces, en particulier dans les multicouches périodiques.

Les méthodes d'analyse des profils ont été décrites dans plusieurs publications [12-14]. Le profil de composition à l' interface aussi bien que la rugosité moyenne sont les deux paramètres qu'il est possible 
d'extraire de ces mesures. Récemment [15], cette méthode a été utilisée sous rayonnement synchrotron en augmentant la sensibilité par une méthode de différence avec un échantillon témoin sur du silicium oxydé. Le calcul des intensités transmises et réfléchies par des interfaces de profils arbitraires est difficile à faire de manière exacte. Il a été amélioré par plusieurs auteurs [16-18] en généralisant l'approximation de Nevot et Croce dans la limite de courtes corrélations latérales. Les approximations employées sont comparées à la solution exacte obtenue pour le profil spécial d'Epstein [18].

La réflectivité étendue s'entend en général pour décrire la réflectivité sur les plans de Bragg au voisinage d'une position de diffraction (par exemple du substrat). Elle peut être modélisée de manière simple dans un modèle cinématique à une dimension [19]. Des interférences entre les différentes couches ayant des distances entre plans diffractants variables apparaissent : la modélisation permet de calculer un profil de distance entre plan ou un profil de composition à la traversée de l'interface (Fig. 3).

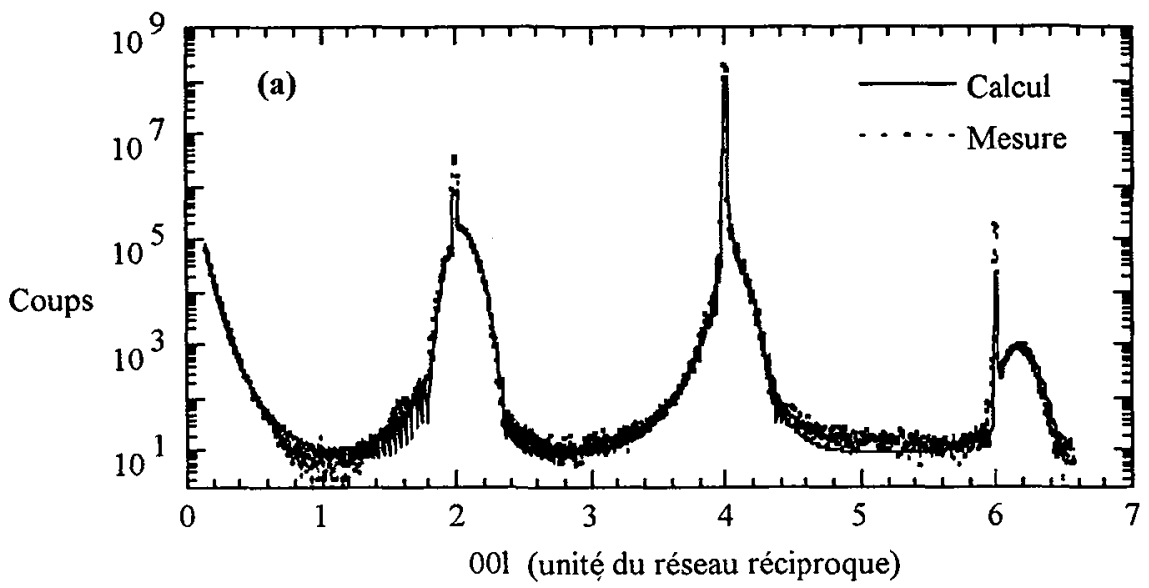

Figure 3 : Profil de réflectivité dans un empilement de CdTe (substrat)/SmTe/CdTe(15 nm). Cette courbe étendue jusqu'aux diffractions 004 et 006 permet de modéliser le profil atomique aux interfaces avec la couche de SmTe (d'après [20]).

Dans le cas de multicouches à puits quantiques périodiques les profils de réflectivité montrent les pics de diffraction de la période de la multicouche. Les profils exacts sont très sensibles aux profils d'interfaces : c'est ainsi qu'il est possible d'extraire une valeur moyenne de la "largeur" de l'interface. Cependant il n'est pas possible par réflectivité simple de déterminer l'origine de cette largeur qui peut aussi bien provenir d'une ondulation de l'interface que d'une interdiffusion des espèces chimiques. Kaganer et al. [21] ont montré que l'intensité des pics de diffraction d'une multicouche est influencée par la rugosité des interfaces. Pour des interfaces parfaites ou de rugosité non corrélée l'intensité est donnée par celle de l'onde stationnaire qui s'installe dans la multicouche, par contre dans le cas de rugosité interfaciale corrélée des effets d'interférence affectent les intensités des pics de la multicouche.

Dans le cas d'interface liquide-solide une modélisation spéciale est nécessaire pour inclure les vibrations de l'interface, les relaxations de surface et l'ordre à courte distance ou longue distance dans le liquide et le solide [22]. Ces études ont été étendues aux interfaces solide-electrolyte [23]. Dans ce cas la mise en ordre des molécules d'eau au voisinage de l'interface a été détectée et surtout la densité des molécules d'eau est remarquablement plus élevée dans la première monocouche d'eau que dans les suivantes.

\subsection{La diffusion diffuse non spéculaire}

La diffusion diffuse (hors spéculaire et hors diffraction) est de plus en plus étudiée car bien que les signaux soient très faibles, les sources synchrotron intenses de la nouvelle génération autorise des mesures dans des temps raisonnables. Ce signal porte en effet une information précieuse sur les rugosité d'interface : les corrélations spatiales des défauts d'interface à la fois entre couches et dans une même interface peuvent être déduites. Plusieurs auteurs ont calculé récemment la distribution dans le réseau réciproque de la diffusion diffuse autour du spéculaire pour différents types de défauts : l'effet de la rugosité d'interface par exemple en introduisant la valeur quadratique moyenne de la rugosité et la distance de corrélation latérale avec des interfaces abruptes ou diffuses. Une bonne description théorique prenant en compte les fonctions de corrélation de la densité électronique (dans le plan de l'interface et hors du plan), et l'effet de réfraction aux interfaces est donnée par divers auteurs dans le cadre de l' approximation des ondes de Born distordues [5- 
24-26]. Ces travaux théoriques encore en développement sont d'un grand intérêt pour une meilleure caractérisation des interfaces et la connaissance des processus de croissance.

La diffusion aux petits angles est un cas particulier de la diffusion diffuse non spéculaire et peut s'appliquer dans certains cas aux interfaces discontinus ou très inhomogènes. L'exploitation quantitative est classique et permet de déterminer des tailles de domaines, d'îlots ou de trous ou de suivre des réactions interfaciales [27].

\subsection{Les méthodes de diffraction}

La méthode de diffraction $\mathrm{X}$ en incidence rasante a fait l'objet de plusieurs revues (voir par exemple [7]). La méthode a d'abord été utilisée pour l'étude des surfaces [28] et a conduit à de très nombreux résultats. Puis elle a été utilisée pour l'étude des interfaces enterrées lorsque celles ci sont périodiques [29-30]. Dans ce cas la périodicité doit être spécifique de l'interface et ne pas se confondre avec celle du substrat ou de la couche déposée. Les interfaces reconstruites ou les joints de grains de coïncidence offrent des exemples de ce cas, les intensités diffractées et donc les facteurs de structure peuvent y être déterminés. Les approches classiques des résolutions de structure sont alors employées. Il faut remarquer que le vecteur de diffraction, $Q$, est en général contenu dans le plan d'interface et dans ce cas seule la projection de la structure atomique de l'interface peut être déterminée. L'exploitation des tiges de troncature perpendiculaires à l'interface, sur les noeuds de diffraction provenant de cette interface, permet d'avoir des vecteurs de diffraction ayant une composante normale au plan et donc d'obtenir des renseignements sur la structure hors du plan de l'interface [31].

De Santis [32] a été le premier à utiliser la diffraction anormale sur une multicouche. Cette méthode permet, par une mesure de part et d'autre du seuil d'absorption d'un élément spécifique, d'être sensible à un élément seulement d'une multicouche.

L'interprétation quantitative est presque toujours cinématique [33], bien que quelques auteurs aient calculé l'effet de la prise en compte de la théorie dynamique sur les tiges de troncature [34]. Cet effet est cependant la plupart du temps négligeable.

\section{LES RÉSULTATS EXPÉRIMENTAUX}

\subsection{Interfaces semiconducteur-semiconducteur}

\subsubsection{Semiconducteur isoélectronique}

Les interfaces dans les semiconducteurs sont en général assez abruptes et stables. Les liaisons étant rigides et de caractère covalent les hétéroépitaxies sont aisées à faire et des reconstructions peuvent avoir lieu à l'interface. Les diffusions dans ces matériaux se font en général à haute température ce qui assure une bonne stabilité thermique. Dans les alliages, par contre, les ségregations de surface limitent la raideur des interfaces.

Les structures de croissance par épitaxie (jets moléculaires ou toutes autre méthodes) ont reçu une forte attention due à leur intérêt pour la microélectronique ou l'optoélectronique. Les structures à puits quantiques à confinement des paires électrons-trous, nécessitent en effet des dépôts successifs de matériaux différents. Les interfaces ainsi créées doivent être caractérisées afin de connaître la rugosité, la distribution latérale des marches, la propagation des marches d'une couche à l'autre dans le cas de dépôt multiple. Dans le cas de matériaux à fort désaccord de maille le problème est d'éviter l'introduction de dislocations ou de tout autre défaut : la caractérisation des relaxations de contraintes apparaissant à l'interface est donc de grande importance. Pour toutes ces raisons les interfaces dans les semiconducteurs, en particulier isoélectronique ont été très étudiées par rayons X. Souvent d'ailleurs ces études sont complétées par l'observation en microscopie électronique qui fournit de précieux renseignements pour l'interprétation des résultats.

Ikarashi et al. [35] ont déterminé, à la résolution atomique, la structure interfaciale de l'interface $\mathrm{Si} / \mathrm{Ge}(100)$ en utilisant la diffraction en incidence rasante. Dans le cas étudié, la couche insérée de Si entre deux couches de Ge contient 5 monocouches. Une surstructure d'interface $2 \times 1$ apparaît. L'exploitation des intensités diffractées, et donc des facteurs de structure pour cette mise en ordre, permet aux auteurs de proposer un modèle atomique contenant un plan (une bicouche) ayant alternativement une paire de Si et une paire de Ge sur l'interface Si sur Ge. Par contre l'interface Ge sur Si est abrupte et sans double périodicité. Cette structure indique que le remplacement atomique durant le dépôt du $\mathrm{Si}$ est du à la ségrégation de surface du Ge. La relaxation optimum des contraintes conduit à la mise en ordre $2 \times 1$ sur la surface de la couche en train de croître. Cette mise en ordre est ensuite enterrée durant le dépôt et ne se reproduit plus lorsque la deuxième interface est créée. 
Dans une étude similaire [36] l'insertion d'une monocouche de Ge dans du Si (100) a été mesurée. La surstructure n'est pas visible mais une étude combinant la diffraction $\mathrm{X}$ et les ondes stationnaires a permis de déterminer les rugosités d'interface et les relaxations du réseau cristallin pour accommoder la monocouche insérée. Les relaxations sont vues comme des translations rigides du réseau perpendiculairement à l'interface. Les valeurs mesurées de translation sont compatibles avec la théorie élastique en ce qui concerne la distance substrat -couche redéposée mais la distance entre la couche de Ge et le Si redéposé correspond plutôt à une longueur de liaison fixe par rapport au cristal massif.

Une autre approche a été adoptée par Tsai et al. [37]. Dans une série d'hétérojonctions de $\mathrm{Si} / \mathrm{SiG̀}$ déposée à une température de $550^{\circ} \mathrm{C}$ par décomposition chimique en phase vapeur, il est nécessaire d'introduire un gradient de composition pour expliquer les courbes de diffraction $\mathrm{X}$ du superréseau. L'épaisseur de la couche de transition est de l'ordre de $1,9 \mathrm{~nm}$ à $550^{\circ} \mathrm{C}$ et décroît avec la température. Les conditions expérimentales conduisent donc dans ce cas à une importante interdiffusion chimique. La composition chimique nominale de l'alliage SiGe n'est donc atteinte qu'au bout d'un certain temps de dépôt.

Beaucoup d'études ont également été faites sur les semiconducteurs III-V en particulier dans les multipuits quantiques, nous ne citerons que les plus récentes [12-13-38-39]. Chang [39], par exemple, a étudié l'effet d'un traitement thermique sur les interfaces d'un multipuits quantique de AlAs/GaAs. Ces multipuits fabriqués à basse température $\left(310^{\circ} \mathrm{C}\right)$ par épitaxie par jets moléculaires sont de bonne qualité Cependant, comme le détecte la diffraction $\mathrm{X}$, la rugosité d'interface et l'interdiffusion augmente rapidement à partir d'une température de $700^{\circ} \mathrm{C}$. La modélisation des profils de diffraction de la multicouche conduit à la détermination des profils chimiques à l'interface et donc à l'énergie effective d'activation du phénomène qui est de $0.24+/-0.07 \mathrm{eV}$. Il est suggéré que l'excès d'arsenic associé à la faible température de croissance conduit directement à un excès de lacunes et donc à une interdiffusion aux interfaces avec une faible énergie apparente de diffusion.

Dans une étude plus ancienne sur des réseaux contraints Clarke et al. [40] ont étudié in-situ la relaxation des contraintes pendant le recuit. L'expérience sous rayonnement synchrotron dispose d'un multidétecteur permettant d'enregistrer l'évolution temporelle durant les recuits thermiques rapides. Les multicouches de As- $\operatorname{In}_{\mathrm{X}} \mathrm{Ga}$ 1-x/GaAs se relâchent par des mouvements brusques qui ont été étudiés en détail.

Benzaquen et al.[41] se sont intéressés à l'effet aux interfaces des interruptions de croissance en

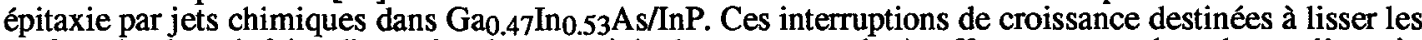
surface (et donc à faire disparaître les rugosités dues aux marches), affectent cependant de manière très sérieuse le profil chimique des interfaces. Ces effets étudiés par diffraction $\mathrm{X}$ sur les multicouches et modélisation correspondantes, conduisent aux résultats suivants : une exposition de la surface de InP à l'arsine pendant $2 \mathrm{~s}$ est suffisante pour créer 3 monocouches de In $\mathrm{As}_{0.55} \mathrm{P}_{0.45}$ ternaire en compression à l'interface. De plus l'exposition de Ga0.47In0.53As à la phosphine pendant 2 s conduit à la formation d'un alliage quaternaire en tension $\mathrm{Ga}_{0.48} \mathrm{In}_{0.52} \mathrm{As} 0.21 \mathrm{P}_{0.79}$. Par contre l'exposition aux hydrures donne des interfaces bien moins désordonnées.

Des études similaires ont été effectuées [42] dans un système modèle : InP/ As et Ga As/P afin de déterminer l'effet de l'incorporation de l'As ou du P respectivement dans InP et GaAs. Les déformations du réseau cristallin détectée par diffraction $\mathrm{X}$ permettent de mesurer les vitesse d'incorporation des éléments en fonction de la température. L'arsenic s'incorpore rapidement alors que le phosphore s'incorpore lentement. Dans cette étude également il a été mis en évidence que $\mathrm{GaP}$ en croissance sur $\mathrm{GaAs}$ à $650^{\circ} \mathrm{C}$ donne une interface à composition intermédiaire GaAs0.5 $\mathrm{P}_{0.5}$ par incorporation d'arsenic.

Une étude plus complète par diffraction et par diffusion hors spéculaire en incidence rasante a été effectuée par Jenichen [43] sur des superréseaux à courte période de InAs/AlSb. La séquence à l'interface est soit $\mathrm{Al} / \mathrm{As}$ soit In/Sb suivant les conditions de dépôt. Les rugosités d'interface dépendent de ce type d'empilement à l'interface. Par ailleurs les distances de corrélation verticale et dans le plan dépendent beaucoup du degré de relaxation du super-réseau. Une très grande corrélation verticale des marches est observée pour les interfaces du type In/Sb.

Pour les structures dont l'épaisseur excède l'épaisseur critique des dislocations apparaissent aux interfaces. L'étude de la diffusion diffuse des rayons $\mathrm{X}$ permet d'obtenir une caractérisation rapide et non destructive du contenu en dislocations [44]. Dans (001) InGaAs/(AlGa)As cette diffusion est sensible à la densité et à l'orientation de ces dislocations. Pour des dislocations le long de [1-10] la diffusion diffuse est confinée perpendiculairement aux défauts. Par contre lorsque le réseau de dislocations est complet avec [110] et [1-10] la diffusion diffuse est presque isotrope. Cette étude a pu se faire en fonction de l'épaisseur du puits de InGaAs.

Enfin une étude cristallographique complète des dislocations d'une interface incohérente a été effectuée sur le système GaAs/GaSb [31]. Les dislocations d'interface forment dans ce cas un réseau ordonné bidimensionnel qui produit des noeuds de diffraction sous forme de satellites des noeuds de diffraction du substrat et de la couche déposée. La mesure des intensités de ces satellites et leur évolution le long des tiges de troncature a permis de modéliser la forme du coeur des dislocations à une échelle atomique. Il a été mis en évidence que l'interface $\mathrm{GaAs} / \mathrm{GaSb}$ n'est pas plane au niveau du coeur avec une tendance à éjecter les 
gros atomes ( $\mathrm{Sb})$ de la zone en compression (Fig. 4). Ces observations ont conduit à proposer un modèle de croissance pour les désaccords de maille important.

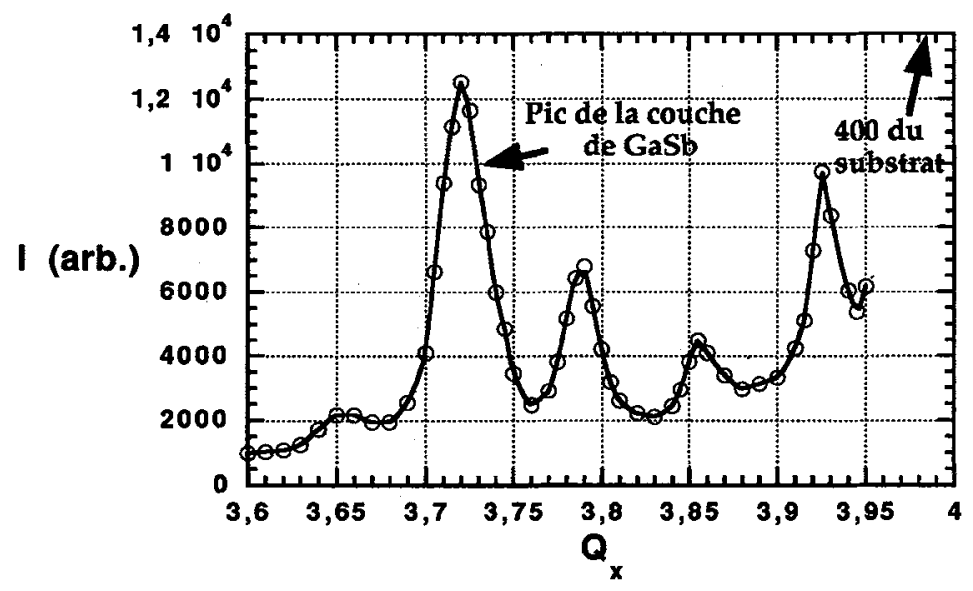

Figure 4 : Etude de l'interface $\mathrm{GaAs}(100) / \mathrm{GaSb}$ par diffraction $\mathrm{X}$ en incidence rasante. Le profil au voisinage de la diffraction 400 du substrat et de la couche déposée fait apparaître quatre satellites dus au réseau périodique de dislocations d'interface (d'après [31])

Les études dans les semiconducteurs II-VI sont moins nombreuses. Citons Kemner [45] qui a étudié l'interface $\mathrm{ZnTe} / \mathrm{CdSe}$ en diffraction $\mathrm{X}$ et en absorption dans les multicouches de ces matériaux. Les mesures font apparaître qu'un échange a lieu à l'interface dans le CdSe : le $\mathrm{Zn}$ vient remplacer le Cd à une interface et le Se vient remplacer le Te à l'autre interface. Des cas plus complexes d'évolution des interfaces dans des matériaux amorphes peuvent être traités dans la mesure où les interfaces sont multiples et périodiques [46]. Les multicouches Se/CdSe amorphes présentent des profils de diffraction en réflectivité étendue qui permettent de suivre l'évolution des rugosités d'interface avec le recuit. Des raidissements du profil des interfaces sont ainsi observés à $360^{\circ} \mathrm{C}$ avec une largeur d'interface décroissant de 1,6 à $1,3 \mathrm{~nm}$. L'origine de ce phénomène reste à préciser.

\subsubsection{Semiconducteur non-isoélectronique}

Les études ont surtout porté sur les hétérocroissances II-VI sur III-V ou III-V sur Si. Mais l'intérêt porté aux plans de dopants ( $\delta$-doping) a également suscité des études de rayons $\mathrm{X}$ intéressantes.

Bourret et Fuoss [30] ont par exemple résolu complètement la projection de la structure d'une hétérojonction incohérente mais commensurable dans le système $\mathrm{GaAs}(001)-\mathrm{CdTe}(111)$. En conjonction avec des observations au microscope électronique les auteurs proposent, à partir de la diffraction X en incidence rasante une structure tridimensionnelle complète de l'interface (Fig. 5). En effet l'interface est périodique avec une surstructure $\sqrt{2}(2 \times 9)$. L'interface se trouve entre un plan de Ga et un de Te. De plus les atomes de Ga situés à l'interface sont presque tous penta-coordonnés avec deux As et 3 Te comme voisins. Il se trouve qu'une telle configuration respecte la neutralité électrique à l'interface et un nombre d'électrons de deux par liaisons.

Dans le même système [47], des mesures de diffraction $X$ à plus longue distance de l'interface montrent que les contraintes résiduelles ne sont pas complètement relâchées avant $20 \mathrm{~nm}$ de l'interface. Ce relâchement a d'ailleurs été attribué à la présence de micromâcles parallèles à l'interface et qui servent de nucléation à la production des dislocations de désaccord. Dans un système équivalent [48], ZnSe sur GaAs (001) une cartographie de la diffusion diffuse étendue au voisinage des pics de diffraction a permis de déduire les profils de contrainte et de mosaïcité à travers les interfaces.

Enfin, l'importance des profilométries de diffraction $\mathrm{X}$ pour l'étude des systèmes $\delta$-dopés a été remarquée [49]. Hart [50] a par exemple mesuré les rugosités d'interface de monoplans d'Al dans $\mathrm{GaAs}$ introduit durant une croissance à $400^{\circ} \mathrm{C}$ ( $\delta$-barrière). L'étude par diffraetométrie $\mathrm{X}$ à haute résolution (réflectivité étendue) sur une multicouche périodique montre que la rugosité est inférieure à deux monocouches. La comparaison avec le $\delta$-dopant Si dans GaAs a été ensuite faite. 


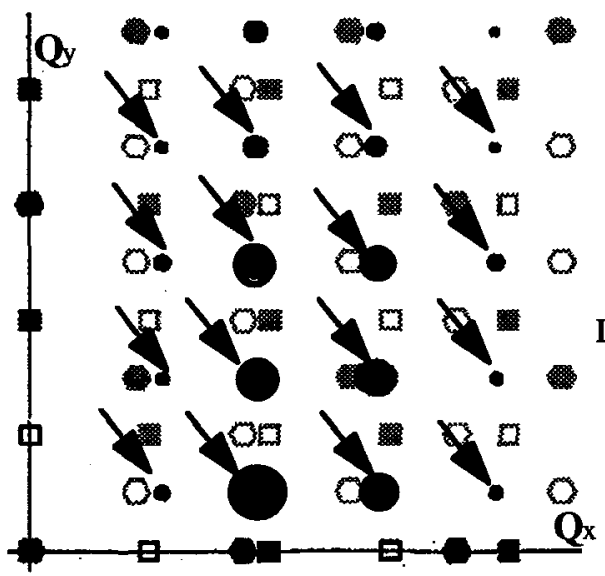

a)

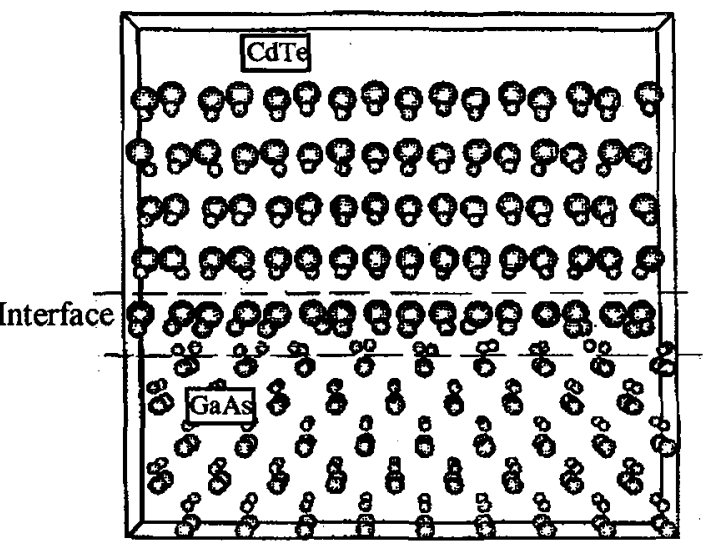

b)

Figure 5 : Etude de l'interface $\mathrm{GaAs}(100) / \mathrm{CdTe}(111)$ par diffraction X en incidence rasante a) Diagramme de diffraction dans le plan de l'interface. Les pics de diffraction de la surstructure produite à l'interface sont marqués d'une flèche et le diamètre est proportionnel à l'intensité mesurée. b) Modélisation de l'interface à trois dimensions déduite des mesures X et des images de microscopie électronique à haute résolution.(extrait de [30])

\subsection{Interfaces semiconducteur-métal}

Pour les mêmes raisons que pour les interfaces semiconducteur-semiconducteur, les interfaces métalsemiconducteur ont été très étudiées. Les prises de contact ohmiques sur les dispositifs ou la production de barrière de Schottky ont une telle importance que la fabrication d'hétérostructures aussi parfaites que possible est un objectif toujours poursuivi. La plupart des études sont des études fondamentales sur des systèmes modèles à base de silicium qui visent à comprendre le type d'accrochage du métal sur le semiconducteur. Le nombre important d'électrons disponible sur le métal et la flexibilité des liaisons métalliques autorise un bon accrochage du métal sans distorsions particulière des atomes du substrat, à moins bien sûr que la liaison soit réactive.

Sur le silicium, l'Ag et le $\mathrm{Pb}$ sont très peu solubles et peuvent donc servir de modèle pour l'obtention d'interface plane et non diffusée. Abaruno [51-52] a regardé le système $\mathrm{Ag} / \mathrm{Si}$ (111) après dépôt de plusieurs couches d'Ag. La reconstruction initiale $7 \times 7$ du silicium se conserve sous le dépôt avec de légères modifications, mais celle ci se transforme en une structure $1 \times 1$ après recuit à $200-250^{\circ} \mathrm{C}$. Il n'y a pas de reconstruction concomitante, par exemple vers la $(\sqrt{3} \times \sqrt{3}) \mathrm{R} 30^{\circ}$ qui pourtant apparaît lorsqu'il y a une monocouche d'argent. Ce résultat important illustre le fait qu'une interface enterrée n'a pas de raisons d'être similaire à une couche absorbée du même élément en surface. La structure de l'interface elle même a été approfondie en mesurant les intensités le long des tiges de troncature. Les interfaces sont abruptes avec une légère interdiffusion après recuit.

Howes [53]et Lucas [54] ont étudié le système Pb sur silicium (111). Comme dans le cas de l'Ag la reconstruction $7 \times 7$ est préservée à froid (85K) par le dépôt, ce qui n'est pas le cas de la reconstruction $\sqrt{3}$. Cette dernière est détruite dès les premières couches déposées. Par ailleurs une transition irréversible se produit à l'interface au cours du chauffage entre $150^{\circ} \mathrm{C}$ et $300^{\circ} \mathrm{C}$ et la reconstruction $7 \times 7$ disparaît. Les mesures de réflectivité spéculaire, aussi bien que le long des tiges de troncature, ont montré que la première couche de plomb était toujours incomplète. Cependant la modélisation des positions atomiques à l'interface elle même est rendue délicate car il n'y a ni une claire corrélation entre les sites des atomes de $\mathrm{Si}$ et de $\mathrm{Pb}$ ni de surstructure ni un complet désordre. Par contre il est nettement établi que la transition entre les sites du $\mathrm{Si}$ . "massif" et ceux du Pb "massif" se fait sur une seule couche.

Les multicouches métal lourd -silicium qui peuvent servir de miroirs ont fait l'objet de très nombreuses observations par rayons $\mathrm{X}$ afin de caractériser les rugosités d'interface et les corrélations entre défauts d'interface. Pour une étude récente voir par exemple [55]. Dans ce travail la réflectivité et la 
diffusion diffuse ont été mesurées sur des multicouches $\mathrm{W}_{1-\mathrm{x}} \mathrm{Si}_{\mathrm{x}} / \mathrm{Si}$. Les résultats ont été interprétés dans le cadre de l'approximation de Born des ondes distordues en prenant en compte les corrélations verticales de la rugosité. L'étude en fonction de l'angle de dépôt a montré que la rugosité augmentait et les corrélations diminuaient avec l'obliquité du dépôt. Par contre la rugosité d'interface est insensible à la composition de l'alliage de tungstène jusqu'à $x=0,67$, alors que les corrélations verticales augmentent d'un ordre de grandeur. Il ne semble pas que l'interface ait un comportement fractal.

Les siliciures forment une classe de conducteur métallique qui a été largement étudiée. Les siliciures de terre rare $\mathrm{ErSi}_{2-\mathrm{x}}$ (x environ 0.3) sur $\mathrm{Si}(111)$ déposés par dépôt réactif en épitaxie sur le silicium se prêtent bien aux études par diffraction en incidence rasante [56]. En effet des lacunes s'introduisent dans les plans de Si du siliciure pour relâcher les contraintes de compression introduites à l'interface. Ces lacunes s'ordonnent et introduisent une surstructure qui apparaît dès que l'interface se forme. Cette reconstruction $(\sqrt{3} \times \sqrt{3}) \mathrm{R} 30^{\circ}$ permet de résoudre la structure atomique en détail au voisinage de l'interface : la position des atomes est identique à celle que l'on trouve dans les films épais de siliciures et la première couche d'Er est localisée sur les sites T4 du substrat. Les sites des 8 premières couches ont pu ainsi être étudiés. Seule la première couche de siliciure montre un ordre à longue distance des lacunes. Dans une étude de réflectivité et diffusion diffuse très détaillée [57] les paramètres des interfaces d'une multicouche $\mathrm{CoSi}_{2} / \mathrm{Si} / \mathrm{CoSi} 2 / \mathrm{Si}(111)$ ont été mesurés. Les interfaces présentent des rugosités de marche de l'ordre d'une monocouche avec une corrélation partielle d'une couche à l'autre jusqu'à la surface de la dernière couche de $\mathrm{CoSi}_{2}$. La fonction de distribution latérale des rugosités de marche a également été précisée. Ces mesures se corrèlent bien avec les mesures de microscopie tunnel et de microscopie électronique.

Boudet [20] a effectué des mesures de réflectivité étendue dans le système $\mathrm{Sm} / \mathrm{CdTe}(001)$. Une couche enterrée de Sm dans CdTe en épitaxie réagit avec le Te pour former des tellurures dont la structure et le mode d'accolement avec l'interface ont été précisés. La distribution de densité électronique le long de l'axe de croissance a été modélisée pour reproduire les résultats expérimentaux. Un gradient de composition sur des distances relativement importante $(2 \mathrm{~nm})$ a du être introduit aux interfaces tellurures/CdTe. L'origine de ce résultat un peu surprenant est, en fait, que l'interface n'est pas plane mais distribuée en chevrons réguliers. Ce résultat n'aurait pu être obtenu par les rayons X seuls, car c'est la microscopie électronique à haute résolution qui a finalement donné la clé de cette structure. Cette étude illustre bien le fait que l'observation des images aussi bien que de la diffraction/diffusion sur le même objet est souvent indispensable.

\subsection{Interfaces semiconducteur-isolant}

De très nombreuses études ont été effectuées sur l'interface $\mathrm{Si} / \mathrm{SiO}_{2}$, car son importance technologique est énorme. Citons par exemple une étude récente de Awaji et al. [15] . Pour des épaisseurs de 4 et $7 \mathrm{~nm}$ d'oxyde ces auteurs ont mis en évidence l'existence d'une fine couche $(1 \mathrm{~nm})$ interfaciale à forte densité $\left(2,4 \mathrm{~g} \mathrm{~cm}^{-3}\right)$. Cette couche varie en épaisseur avec le type de traitement d'oxydation.

Par ailleurs Lucas et al. [58] ont étudié l'interface $\mathrm{CaF}_{2} / \mathrm{Si}$ (111) par diffraction et mesure des tiges de troncature. Ces auteurs ont mis en évidence le site de $\mathrm{Ca}$ sur le silicium : le site T4 est occupé. De plus une transition de phase à l'interface vers une surstructure ordonnée $(\sqrt{3} \times \sqrt{3}) \mathrm{R} 30^{\circ}$ apparaît de manière incommensurable avec le substrat.

\subsection{Interfaces métal-métal}

La détermination des rugosités d'interface dans les multicouches métalliques est un paramètre très important. En effet cette rugosité détermine les performances des structures, par exemple la magnéto résistance géante. Or les interfaces sont très sensibles aux conditions de fabrication car les interdiffusions sont faciles dans les métaux.

Nawate [59] a par exemple essayé de déterminer si les changements de magnéto résistance dans les multicouches $\mathrm{Co} / \mathrm{Cu}$ après un recuit à $200^{\circ} \mathrm{C}$ affectait la rugosité d'interface. En fait les changements vus en rayons $\mathrm{X}$ sont très faibles et la dégradation des propriétés magnétiques est attribuée à des réarrangements très locaux dont les déplacements moyens vus par rayons $X$ sont insignifiants. Heyvaert [60] a étudié les multicouches $\mathrm{Ag} / \mathrm{Au}$ et $\mathrm{Cu} / \mathrm{Au}$ par réflectivité à petit et grand angle (réflectivité étendue). La rugosité moyenne des interfaces qui est déduite de ces mesures est comparée à la rugosité de la surface telle qu'elle est mesurée in-situ par microscopie tunnel. Le domaine de distance spatiale qui fait correspondre les deux résultats est celui de la taille moyenne des grains, ce qui s'explique aisément. La complémentarité des deux méthodes est ici illustrée car la rugosité effective vue par les rayons $\mathrm{X}$ ne recouvre pas tout le spectre de distance spatiale et se trouve limité par la taille des grains dans cet exemple. Dans le cas de terres rares magnétiques Mozley [61] a effectué des mesures de diffraction X in-situ sur le système $\mathrm{Gd} / \mathrm{Mo}(110)$ : à $300 \mathrm{~K}$ la variation de la réflectivité est compatible avec une croissance $2 \mathrm{D}$ pour la première monocouche mais 
celle ci devient rapidement tridimensionelle. Par contre une amélioration très nette de la croissance 2D est observée après exposition à l'oxygène. De Santis et al. [32] ont mesuré le profil du Fer dans des multicouches $\mathrm{Fe} / \mathrm{Ru}$ déposées par jets moléculaires et dans laquelle le Fer est dans une phase métastable hexagonale.

Plusieurs études récentes ont été aussi effectuées sur les métaux solides en équilibre avec leur phase liquide [62]. Ces auteurs ont mis en évidence une stratification périodique à l'échelle atomique dans le liquide au voisinage de l'interface solide-liquide. Cette mise en ordre décrốt rapidement à partir de l'interface, elle est de 0,6 $\mathrm{nm}$ de longueur caractéristique dans le $\mathrm{Ga}$. Cette valeur est à comparer avec la valeur déjà trouvée dans une étude similaire sur le mercure [63] qui est de l'ordre de $0,3 \mathrm{~nm}$. La rigidité plus grande des liaisons dans le gallium pourrait expliquer cette différence (ou les phénomènes de surfusion)

\subsection{Interfaces métal-oxyde}

Malgré leur importance, tant au niveau fondamental qu'appliqué, ce type d'interface a été relativement peu étudié par rayons $\mathrm{X}$. Les liaisons atomiques entre ces deux types de matériaux ( grand gap d'un coté et énergie de plasmon faible de l'autre) sont en général relativement faible et leur origine est mal connue. L'importance pratique des revêtements métalliques sur les verres ou à l'inverse la protection de pièces métalliques par des films céramiques est cependant suffisante pour que ce sujet préoccupe vivement les laboratoires. La microscopie électronique est la méthode la plus utilisée actuellement sur ces systèmes.

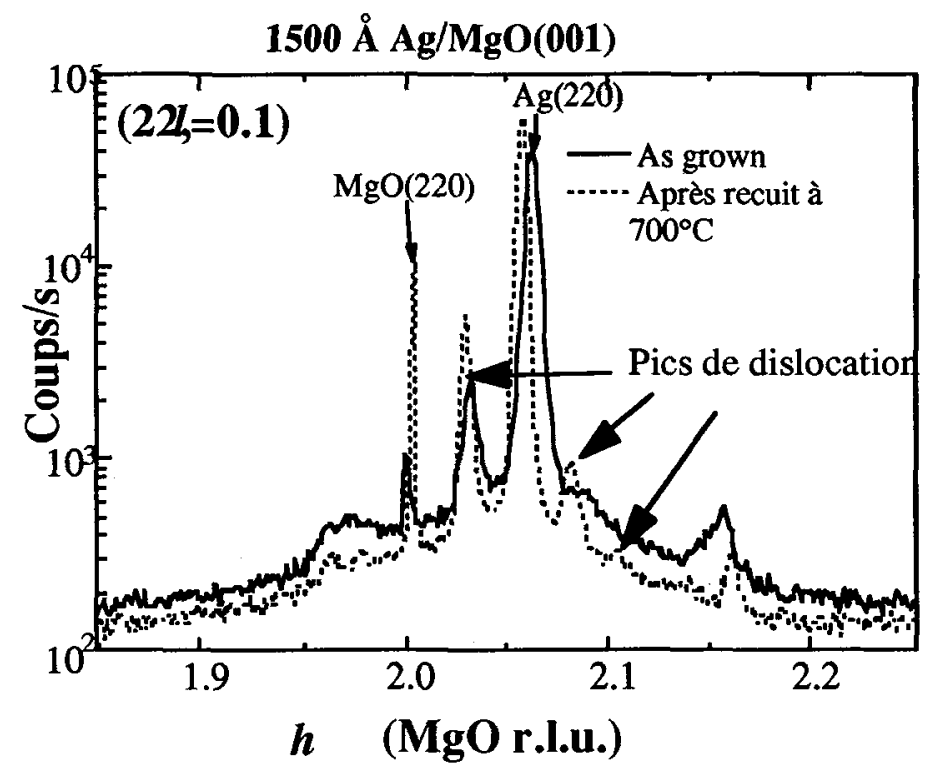

Figure 6 : Evolution des pics de diffraction dus aux réseaux de dislocations dans Ag/MgO en fonction du recuit (d'après [64]

Dans le domaine des rayons $\mathrm{X}$ des systèmes modeles comme $\mathrm{Ag} / \mathrm{MgO}(100)$ ont été choisis car les relations d'épitaxie y sont simples, le désaccord de maille est faible et les transferts de charge faibles [64]. Ces auteurs ont montré qu'un réseau de dislocation régulier s'établit à l'interface pour accommoder l'écart de maille : celui ci donne des pics de diffraction dus à la surstructure périodique qui s'établit ainsi. L'analyse des conditions d'extinction de ces pics permet de déterminer les paramètres géométriques de ces dislocations. L'orientation $<110>$ des dislocations n'est pas celle observée en microscopie électronique ce qui s'explique probablement par des températures de dépôt différentes. L'évolution du réseau de dislocations avec la température de recuit a été suivie (Fig. 6)

Dans un travail récent [65] la croissance in-situ de l'Ag/MgO par diffraction $\mathrm{X}$ en incidence rasante a été suivie. La croissance depuis les fractions de monocouche jusqu'à des épaisseurs de $60 \mathrm{~nm}$ dépend peu des défauts de la surface (marches). La croissance a lieu en îlots. Des macles apparaissent dès le début du dépôt tandis que des fautes d'empilement sont présentes dès que la coalescence a lieu. Les mécanismes de 
relaxation des contraintes sont précisés : les dislocations s'introduisent à l'interface par les côtés des îlots après une violente déformation sur les bords de ces îlots. Ce mécanisme est continu êt débute bien avant la coalescence.

\section{CONCLUSION}

L'étude des interfaces enterrées par les rayons $\mathrm{X}$ a déjà donné de nombreux résultats. Les méthodes de laboratoire utilisant une anode toumante sont maintenant largement implantées et sont devenues des méthodes de caractérisation systématique, en particulier la réflectivité (y compris la réflectivité étendue) ou les profils de diffraction à haute résolution. Les outils mathématiques d'exploitation des résultats sont également bien maîtrisés. Cet ensemble d'outils a permis une caractérisation des profils d'interface et de leur rugosité sans équivalent par d'autres méthodes. Il reste qu'il est toujours aussi difficile de distinguer un profil chimique d'un profil rugueux (dont l'interface oscille). Des progrès récents.ont été faits dans ce domaine par l'analyse du diffusé hors spéculaire. La mesure des corrélations dans le plan des couches ou perpendiculairement ouvre là des perspectives intéressantes. Cependant les outils d'exploitation sont plus complexes et dépendent souvent du modèle initial choisi, il y a donc aussi des progrès à attendre dans le domaine théorique.

Enfin l'arrivée et le développement des sources de rayonnement synchrotron rendent plus accessibles les méthodes de diffraction aussi bien que l'analyse du diffusé hors spéculaire. Les flux étant nettement plus intense la résolution spatiale dans toutes les directions est bien meilleure et de nouveaux résultats devraient apparaître sur les interfaces dans un avenir proche.

On ne saurait cependant trop insister sur l'importance qu'il y a à combiner plusieurs techniques sur le même échantillon, en particulier les méthodes d'imagerie directe (microscopie électronique et microscopie tunnel ) avec les techniques qui utilisent le réseau réciproque. Ces dernières donnent une information moyennée sur l'ensemble de l'échantillon et ont souvent besoin d'une vision plus localisée pour éviter les erreurs d'intérprétation. Cela est d'autant plus vrai que la méthode nécessite des outils d'interprétation complexe ou qu'une modélisation initiale est nécessaire.

\section{Références}

[1] Fuoss P. H., et al., Phys. Rev. Lett. 69 (1992) 2791.

[2] Norris C., Phil. Trans. Roy. Soc. A 344 (1993) 557-66.

[3] Kisker D. W., Stephenson G. B., Fuoss P. H. and Brennan S., J. of Crystal Growth 146 (1995) 104-111.

[4] Chen H. D., Mat. Chem.and Phys. 43 (1996) 116-125.

[5] Dietrich S. and Haase A., Phys. Rep. - Review Section of Phys. Letters 260 (1995) 1-138.

[6] Renaud G., J. Phys. III France 4 (1994) 1795.

[7] Vlieg E., Lohmeier M. and Vandervegt H. A., Nucl. Ins. Meth. B 97 (1995) 358-363.

[8] Tanner B. K. and Bowen D. K., J. of Crystal Growth 126 (1993) 1-18.

[9] Roberts K. J., J. of Crystal Growth 130 (1993) 657-681.

[10] Brunel M., Annales de Chimie - Science Des Materiaux 19 (1994) 415-420.

[11] Parrat L. G., Phys. Rev. 95 (1954) 359.

[12] Auvray P., Baudet M., Caulet J. and Regreny A., Revue Phys. Appl. 24 (1989) 711.

[13] Bensoussan S., Malgrange C., Sauvage-Simkin M., N'Guessan K. and Gibart P., J. Appl. Cryst. 20 (1987) 222.

[14] Lu J. R., Lee E. M. and Thomas R. K., Acta Cryst. A 52 (1996) 11-41.

[15] Awaji N., et al., Jap.J. of Appl. Phys.-Letters 35 (1996) L67-L70.

[16] Stearns D. G., J. of Appl. Physics 65 (1989) 491.

[17] Stepanov S. A. and Köhler R., J. of Appl. Physics 76 (1994) 7809.

[18] Caticha A., Phys. Rev. B 52 (1995) 9214-9223.

[19] Robinson I. K., Tung R. T. and Feidenhans'l R., Phys. Rev. B 38 (1988) 3632.

[20] Boudet N., et al., Surf. Sci. Lett. 327 (1995) L515-L520.

[21] Kaganer V. M., Stepanov S. A. and Kohler R., Phys. Rev. B 52 (1995) 16369-16372.

[22] Makov G. and Kornyshev A. A., J. of Chem. Phys. 104 (1996) 1693-1698.

[23] Toney M. F., et al., Surf. Sci. 335 (1995) 326-332.

[24] Holy V., Baumbach T. and Bessiere M., J. of Physics D 28 (1995) A220-A226.

[25] Holy V. and Baumbach T., Physical Review B 49 (1994) 10688.

[26] Deboer D. K. G., Phys. Rev. B 53 (1996) 6048-6064.

[27] Novet T., Kevan S. and Johnson D. C., Mat. Sci. and Eng. 195 (1995) 21-27.

[28] Robinson I. K., Phys. Rev. Lett. 50 (1983) 1145.

[29] Fitzsimmons M. R. and Sass S. L., Acta Metall. 36 (1988) 3103-3122. 
[30] Bourret A., Fuoss P., Feuillet G. and Tatarenko S., Phys. Rev. Lett. 70 (1993) 311.

[31] Bourret A. and Fuoss P. H., Appl. Phys. Lett. 61 (1992) 1034-1036.

[32] de Santis M., et al., Phys. Rev. B 46 (1992) 15465.

[33] Brunel M. and de Bergevin F., Acta Cryst. A42 (1986) 299-303.

[34] Gau T. S. and Chang S. L., Acta Cryst. A 51 (1995) 920-931.

[35] Ikarashi N., Akimoto K., Tatsumi T. and Ishida K., Phys. Rev. Lett. 72 (1994) 3198-3201.

[36] Takahasi M., et al., Jap. J. of Appl. Phys. 34 (1995) 2278-2283.

[37] Tsai W. C., et al., Appl. Phys. Lett. 67 (1995) 1092-1094.

[38] Ferrari C. and Franzosi P., J. of Appl. Physics 65 (1989) 1544.

[39] Chang J. C. P., et al., Appl. Phys. Lett. 67 (1995) 3491-3493.

[40] Clarke R., Dos Passos W., Lowe W., Rodricks B. and Brizard C., Phys. Rev. Lett. 66 (1991) 317-320.

[41] Benzaquen R., Roth A. P. and Leonelli R., J. of Appl. Physics 79 (1996) 2640-2648.

[42] Clawson A. R. and Hanson C. M., J. of Electronic Mat. 25 (1996) 739-744.

[43] Jenichen B., Stepanov S. A., Brar B. and Kroemer H., J. of Appl. Physics 79 (1996) 120-124.

[44] Goorsky M. S., Meshkinpour M., Streit D. C. and Block T. R., J. of Physics D 28 (1995) A92A96.

[45] Kemner K. M., et al., Phys. Rev. B 50 (1994) 14327-14335.

[46] Vateva E. and Nesheva D., J. of Non - Crystalline Solids 191 (1995) 205-208.

[47] Angelo J. E., Gerberich W. W., Bratina G., Sorba L. and Franciosi A., Thin Solid Films 271 (1995) 117-121.

[48] Heinke H., Möller M. O., Hommel D. and Landwehr G., J. of Crystal Growth 135 (1994) 41-52.

[49] Ploog K. H. and Daweritz L., Jap. J. of Appl. Phys. 34 (1995) 691-699.

[50] Hart L., Fewster P. F., Ashwin M. J., Fahy M. R. and Newman R. C., J. of Physics D 28 (1995) A154-A158.

[51] Aburano R. D., et al., Phys. Rev. B 52 (1995) 1839-1847.

[52] Aburano R. D., et al., Surf. Sci. 339 (1995) L891-L896.

[53] Howes P. B., et al., Surf. Sci. A 333 (1995) 646-650.

[54] Lucas C. A. and Loretto D., Surf. Sci. 344 (1995) L1219-L1225.

[55] Jergel M., Holy V., Majkova E., Luby S. and Senderak R.; J. of Physics D 28 (1995) A241-A245.

[56] Lohmeier M., et al., Surf. Sci. 345 (1996) 247-260.

[57] Stettner J., et al., Phys. Rev, B $\mathbf{5 3}$ (1996) 1398-1412.

[58] Lucas C. A., Loretto D. and Wong G. C. L., Phys. Rev. B 50 (1994) 14340-14353.

[59] Nawate M., Inage K., Imada R., Itogawa M. and Honda S., Jap. J. of Appl. Phys. 34 (1995) 3082-3087.

[60] Heyvaert I., Temst K., Vanhaesendonck C. and Bruynseraede Y., J. of Vac. Sci.\& Tech. B B 14 (1996) 1121-1125.

[61] Mozley S., et al., Surf. Sci. B 333 (1995) 961-964.

[62] Regan M. J., et al., Phys. Rev. Lett. 75 (1995) 2498-2501.

[63] Magnussen O. M., et al., Phys. Rev. Lett. 74 (1995) 4444.

[64] Guenard P., Renaud G., Villette B., Yang M. H. and Flynn C. P., Scripta Mett. Mat. 31 (1994) 121.

[65] Robach O., Renaud G., Barbier A. and Guenard P., Surface Review and Letters (1996) à paraître. 